\title{
Generating Two Independent Rotating Magnetic Fields with a Single Magnetic Dipole for the Propulsion of Untethered Magnetic Devices
}

\author{
Nathan D. Nelson and Jake J. Abbott
}

\begin{abstract}
Untethered magnetic devices (UMDs) driven by rotating magnetic fields have been proposed for applications in minimally invasive medicine. Previous work has explored the propulsion and guidance of a single UMD using a rotating magnet dipole source, and actuation of multiple UMDs has focused on the use of uniform magnetic fields. This work seeks to extend the methods for remote actuation using a single magnet dipole source to generate two rotating magnetic fields with arbitrary independent rotation axes at any two points with a common rotation frequency. The solutions found show that there are multiple positions and rotation axes of the magnet dipole that can generate these rotating fields. The method is demonstrated with two screw-type UMDs propelled independently within an agar gel block using a single permanent-magnet actuator.
\end{abstract}

\section{INTRODUCTION}

Robotic technologies have long been attractive for use in minimally invasive medicine. In addition to robotic surgical systems that augment surgical skills, there has been fruitful research in development of microscale and mesoscale in vivo robotic devices that could locally apply medical interventions [1], including targeted drug delivery and localized ablation of tumors. Many of the untethered robotic devices that have been developed have utilized magnetic fields for propulsion and guidance, since magnetic methods enable remote operation without the need for on-board power storage. These untethered magnetic devices (UMDs) have taken on different forms such as chiral swimmers in fluid [2]-[4], screws in soft tissue [5]-[7], and capsule endoscopes in the gastrointestinal tract [8], [9]. We use the term UMD, as opposed to "microrobot", both to indicate that the methods being discussed here do not require the device to be microscale, and to indicate that no intelligence is assumed on-board the device.

The use of rotating magnetic fields as a method of actuation for these and other types of UMD has been particularly attractive, and has been explored in great detail, since it utilizes the strength of the magnetic field rather than the field gradient, which tends to have desirable scaling properties [10]. Previous work exploring different methods of generating rotating magnetic fields for remote actuation includes generating uniform rotating magnetic fields within sets of Helmholtz coils [5], the dipole field of a single rotating permanent magnet [11] or a computer-controlled electromagnetic source [12], or the combined dipole fields of multiple rotating permanent magnets [13].

This work is supported by the National Science Foundation under Grant Nos. 0952718 and 1435827

The authors are with the Department of Mechanical Engineering, University of Utah, UT 84112, USA. \{nathan.d.nelson, jake.abbott\}@utah.edu

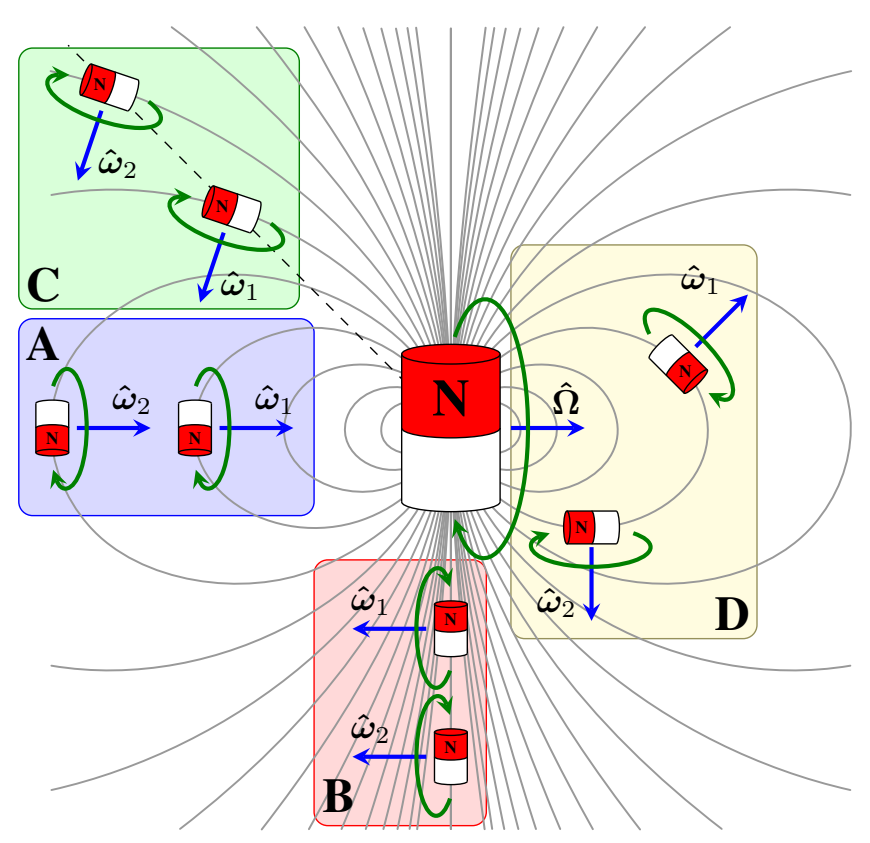

Fig. 1. Examples of four special cases for generation of two independent rotating magnetic fields using a single rotating magnetic dipole. Box A: "axial" positions. Box B: "radial" positions. Box C: general case of $\hat{\omega}_{1}=$ $\hat{\boldsymbol{\omega}}_{2}$. Box D: $\hat{\boldsymbol{\omega}}_{1} \neq \hat{\boldsymbol{\omega}}_{2}$ but coplanar with $\hat{\boldsymbol{\Omega}}$

Mahoney and Abbott [11] have shown that it is possible to generate a rotating magnetic field about any desired axis of rotation at any location in space using a single rotating magnetic dipole located at any other location in space, with a unique location-specific dipole axis of rotation. Since the rotating dipole can be placed anywhere in 3$\mathrm{D}$ space to achieve a given desired rotating-magnetic-field axis, it may be possible to add up to three additional constraints to the problem and still find a solution to achieve the desired rotating-magnetic-field axis. This motivates the fundamental conjecture explored in this paper: Can a rotating magnetic field be generated at two different locations with independent axes of rotation using a single rotating dipole? Such a capability could enable simultaneous independent control of two UMDs (Fig. 1). In this paper, we describe a method to find a set of appropriate locations and rotation axes of a single rotating magnetic dipole that will generate independent rotating magnetic fields at any two locations.

Other works have contributed to the area of multidevice control when using rotating magnetic fields. Methods that rely on rotating uniform magnetic fields necessitate each agent to repond differently to the same global signal. Com- 
mon approaches previously used include heterogeneous device designs that differ in magnetic response to the applied rotating magnetic fields [14], [15], or that respond differently within the fluid [16], [17] or soft-tissue [5] environment in which the UMDs operate. To date, there has been no approach that has exploited the nonuniformity of the magnetic dipole field in generating independent rotating magnetic fields for remote actuation of multiple UMDs.

\section{THEORY}

We seek to find the position $\mathbf{r}_{m}$ and rotation axis $\hat{\Omega}$ for a rotating magnet dipole (i.e., an actuator magnet) that will generate a rotating magnetic field with rotation axis $\hat{\boldsymbol{\omega}}_{1}$ at a location $\mathbf{r}_{1}$, and that will simultaneously generate a rotating magnetic field with rotation axis $\hat{\boldsymbol{\omega}}_{2}$ at a location $\mathbf{r}_{2}$. Note that we use the "hat" notation to designate a unit vector. It is understood that the period of the two generated rotating fields will be same as the period of the rotating magnetic dipole. We will first review the relevant prior results. We shall then approach our problem statement by first exploring how previous methods for generating rotating magnetic fields at a single location using a rotating magnetic dipole may be used to handle the special case when $\hat{\boldsymbol{\omega}}_{1}$ is equal to $\hat{\boldsymbol{\omega}}_{2}$. We will then generalize the approach.

\section{A. Review of Relevant Prior Results}

Fountain et al. [18] showed that when a magnetic dipole $\mathbf{M}\left\{\mathrm{A} \cdot \mathrm{m}^{2}\right\}$ is rotated about an axis $\hat{\Omega}$, with $\mathbf{M}$ orthogonal to $\hat{\Omega}$, there are two regions of space in which the magnetic field generated at a given point also rotates around and orthogonal to an axis, $\hat{\boldsymbol{\omega}}$, where $\hat{\boldsymbol{\omega}}$ is parallel with $\hat{\Omega}$. The first region is the line defined by the location of $\mathbf{M}$ and the vector $\hat{\Omega}$, extending on both sides of the rotating dipole, which were dubbed the "axial" positions (Fig. 1A). In this region, $\hat{\Omega}$ and $\hat{\boldsymbol{\omega}}$ are parallel and collinear, and oriented in the same direction (i.e., $\hat{\boldsymbol{\omega}}=\hat{\boldsymbol{\Omega}}$ ). The second region is the plane defined by the location of $\mathbf{M}$ and the vector $\hat{\Omega}$ (i.e., the plane orthogonal to $\hat{\Omega}$ ), which were dubbed the "radial" positions (Fig. 1B). In this region, $\hat{\boldsymbol{\Omega}}$ and $\hat{\boldsymbol{\omega}}$ are parallel but not collinear, and oppositely oriented (i.e., $\hat{\boldsymbol{\omega}}=-\hat{\mathbf{\Omega}}$ ).

Mahoney and Abbott [11] generalized the method described in [18] to consider all points in space relative to the rotating dipole. Given a magnetic dipole moment $\mathbf{M}$, the magnetic field $\mathbf{H}\left\{\mathrm{A} \cdot \mathrm{m}^{-1}\right\}$ at a point $\mathbf{p}\{\mathrm{m}\}$ measured relative to the position of the magnetic dipole is

$$
\mathbf{H}=\frac{1}{4 \pi\|\mathbf{p}\|^{3}}\left(3 \hat{\mathbf{p}} \hat{\mathbf{p}}^{\top}-\mathbb{I}\right) \mathbf{M}=\frac{1}{4 \pi\|\mathbf{p}\|^{3}} \mathbb{H} \mathbf{M},
$$

where $\hat{\mathbf{p}}$ is the unit vector in the direction of $\mathbf{p}$, and $\mathbb{I}$ is the $3 \times 3$ identity matrix. This is the point-dipole model, which accurately describes the field generated by a spherical permanent magnet, and can be a good approximation for the field generated by well-designed permanent magnets [19] and electromagnets [12]. If $\hat{\mathbf{p}}$ is given, then for a desired rotating magnetic field with rotation axis $\hat{\omega}$, the unique required rotation axis of the dipole can always be calculated as

$$
\hat{\boldsymbol{\Omega}}=\frac{\mathbb{H} \hat{\boldsymbol{\omega}}}{\|\mathbb{H} \hat{\boldsymbol{\omega}}\|}=\frac{3 \hat{\mathbf{p}} \cdot \hat{\boldsymbol{\omega}}}{\|\mathbb{H} \hat{\boldsymbol{\omega}}\|} \hat{\mathbf{p}}-\frac{1}{\|\mathbb{H} \hat{\boldsymbol{\omega}}\|} \hat{\boldsymbol{\omega}}
$$

Alternatively, if $\hat{\mathbf{p}}$ and $\hat{\Omega}$ are both given, the resulting axis of the rotating magnetic field is

$$
\hat{\boldsymbol{\omega}}=\frac{\mathbb{H}^{-1} \hat{\mathbf{\Omega}}}{\left\|\mathbb{H}^{-1} \hat{\mathbf{\Omega}}\right\|}=\frac{(\mathbb{H}-\mathbb{I}) \hat{\mathbf{\Omega}}}{\|(\mathbb{H}-\mathbb{I}) \hat{\mathbf{\Omega}}\|} .
$$

Note that $\|\mathbf{p}\|$ affects the magnitude of $\mathbf{H}$, but does not affect direction of $\mathbf{H}$, nor does it affect the direction of $\hat{\mathbf{\Omega}}$. Another consequence of (2) is that $\hat{\mathbf{p}}, \hat{\boldsymbol{\omega}}$, and $\hat{\boldsymbol{\Omega}}$ are coplanar.

Mahoney and Abbott [11] have also demonstrated that, if $\hat{\Omega}$ and the desired rotating magnetic field rotation axis $\hat{\omega}$ are both given, then the required unit-norm position vector $\hat{\mathbf{p}}$ is found as follows. Let $\rho=\hat{\omega} \cdot \hat{\Omega}$. Then the square of the projection $\gamma=\hat{\mathbf{p}} \cdot \hat{\boldsymbol{\omega}}$ is

$$
\gamma_{ \pm}^{2}=\frac{\rho^{2}+2 \pm \sqrt{8 \rho^{2}+\rho^{4}}}{6}
$$

where the choice of $\gamma_{ \pm}^{2}$ is chosen based on the sign of $\rho$ :

$$
\gamma^{2}= \begin{cases}\gamma_{+}^{2} & \rho \geq 0 \\ \gamma_{-}^{2} & \rho \leq 0\end{cases}
$$

The vector $\hat{\mathbf{p}}$ is then found as

$$
\hat{\mathbf{p}}=|\gamma| \hat{\boldsymbol{\omega}}+\sqrt{1-|\gamma|^{2}} \hat{\boldsymbol{\omega}}_{\perp}
$$

where

$$
\hat{\boldsymbol{\omega}}_{\perp}=\frac{\left(\mathbb{I}-\hat{\omega} \hat{\boldsymbol{\omega}}^{\top}\right) \hat{\Omega}}{\left\|\left(\mathbb{I}-\hat{\omega} \hat{\boldsymbol{\omega}}^{\top}\right) \hat{\Omega}\right\|}
$$

The negative of the solution in (5) is also a valid solution for $\hat{\mathbf{p}}$, if the direction of $\hat{\boldsymbol{\Omega}}$ can be reversed (which is typical of systems designed to generate rotating magnetic dipoles).

B. $\hat{\boldsymbol{\omega}}_{1}=\hat{\boldsymbol{\omega}}_{2}$

The prior results of Mahoney and Abbott [11] can be extrapolated directly for the case when $\hat{\omega}_{1}$ and $\hat{\omega}_{2}$ are equal. For the case when $\hat{\boldsymbol{\omega}}_{1}$ and $\hat{\boldsymbol{\omega}}_{2}$ are equal, and collinear to each other and to the displacement vector between them, $\mathbf{d}=\mathbf{r}_{2}-\mathbf{r}_{1}$, then a magnet dipole position $\mathbf{r}_{m}$ at any other point along the same line in space and with a rotation axis $\hat{\Omega}$ equal to $\hat{\boldsymbol{\omega}}_{1}$ and $\hat{\boldsymbol{\omega}}_{2}$ will generate the desired rotation axes $\hat{\boldsymbol{\omega}}_{1}$ and $\hat{\boldsymbol{\omega}}_{2}$ at the respective desired locations, as depicted in Fig. 1A.

For the case when $\hat{\boldsymbol{\omega}}_{1}$ and $\hat{\boldsymbol{\omega}}_{2}$ are equal and orthogonal to their displacement vector $\mathbf{d}$, then a dipole position $\mathbf{r}_{m}$ at any other point in the plane defined by the normal $\hat{\boldsymbol{\omega}}_{1}\left(\right.$ or $\left.\hat{\boldsymbol{\omega}}_{2}\right)$ and containing both the points $\mathbf{r}_{1}$ and $\mathbf{r}_{2}$ ), and with rotation axis $\hat{\Omega}$ parallel to $\hat{\boldsymbol{\omega}}_{1}$ and $\hat{\boldsymbol{\omega}}_{2}$ but with opposite orientation, will generate the desired rotation axes $\hat{\boldsymbol{\omega}}_{1}$ and $\hat{\boldsymbol{\omega}}_{2}$ at the respective desired locations, as depicted in Fig. 1B.

For the more general case when $\hat{\omega}_{1}$ and $\hat{\omega}_{2}$ are equal, but are neither parallel nor orthogonal to their displacement vector $\mathbf{d}, \hat{\mathbf{p}}$ is still parallel to $\mathbf{d}$, as depicted in Fig. 1C. A dipole position $\mathbf{r}_{m}$ along the line in space defined by $\boldsymbol{L}(s)=\mathbf{r}_{1}+s \mathbf{d}$, where $s$ parameterizes points on the line, 
and with the rotation axis $\hat{\Omega}$ found using (2), will generate the desired rotation axes $\hat{\omega}_{1}$ and $\hat{\omega}_{2}$ at the respective desired locations. Thus, in this generalization of "axial" and "radial" positions, in which the three locations $\mathbf{r}_{1}, \mathbf{r}_{2}$, and $\mathbf{r}_{m}$ are all collinear, and in which $\hat{\mathbf{p}}$ is parallel to $\mathbf{d}$, the resulting rotating-magnetic-field axes of rotation will be equal. Note that, although this solution is a generalization for the case where $\hat{\omega}_{1}=\hat{\omega}_{2}$, it is overly restrictive in the second special case described above and depicted in Fig. 1B; in that case, $L(s)$ only provides a single line in what is actually a plane of possible solutions.

In the solutions described above, we are left with one degree of freedom (i.e., sliding $\mathbf{r}_{m}$ along the line of solutions) to choose $\mathbf{r}_{m}$ based on some additional optimization criterion. For example, $\mathbf{r}_{m}$ can be chosen to avoid collisions of the magnetic-dipole source with objects in the workspace (e.g., a patient), and can be chosen based on the desired magnitude of the rotating magnetic field at the two locations of interest.

\section{C. $\hat{\omega}_{1} \neq \hat{\omega}_{2}$}

We now generalize to examine when $\hat{\omega}_{1} \neq \hat{\omega}_{2}$. We note that there are two cases that require consideration: the case when the three vectors $\hat{\boldsymbol{\omega}}_{1}, \hat{\boldsymbol{\omega}}_{2}$, and $\mathbf{d}$ are all coplanar, and the case when they are not.

1) $\hat{\boldsymbol{\omega}}_{1}, \hat{\boldsymbol{\omega}}_{2}$, and $\mathbf{d}$ coplanar: We begin by considering a special case where $\hat{\omega}_{1}$ and $\hat{\omega}_{2}$ are not equal but are coplanar with their displacement vector $\mathbf{d}$. Note that this also includes the case where $\hat{\boldsymbol{\omega}}_{1}$ and $\hat{\boldsymbol{\omega}}_{2}$ are parallel and oriented in opposite directions (i.e., $\hat{\boldsymbol{\omega}}_{1}=-\hat{\boldsymbol{\omega}}_{2}$ ). Neither $\mathbf{r}_{m}$ nor $\hat{\Omega}$ is known in general, however, and lack of knowledge of $\mathbf{r}_{m}$ implies a lack of knowledge of $\hat{\mathbf{p}}_{1}$ and $\hat{\mathbf{p}}_{2}$. From (2), we know that $\hat{\boldsymbol{\omega}}_{1}, \hat{\mathbf{p}}_{1}$, and $\hat{\boldsymbol{\Omega}}$ must be coplanar, and that $\hat{\boldsymbol{\omega}}_{2}, \hat{\mathbf{p}}_{2}$, and $\hat{\boldsymbol{\Omega}}$ must be coplanar, so an assumed $\hat{\boldsymbol{\Omega}}$ that is also in the plane with $\hat{\boldsymbol{\omega}}_{1}, \hat{\boldsymbol{\omega}}_{2}$, and $\mathbf{d}$ will enable calculation, using (4)-(6), of a vector $\hat{\mathbf{p}}_{1}$ for position $\mathbf{r}_{1}$ and a vector $\hat{\mathbf{p}}_{2}$ for position $\mathbf{r}_{2}$. The dipole position, $\mathbf{r}_{m}$, at the intersection of the line extended from $\mathbf{r}_{1}$ in the direction of $-\hat{\mathbf{p}}_{1}$ and the line extended from $\mathbf{r}_{2}$ in the direction of $-\hat{\mathbf{p}}_{2}$ with the assumed rotation axis $\hat{\Omega}$ will generate the desired rotation axes at the desired positions, as depicted in Fig. 1D. Note that any assumed $\hat{\Omega}$ that is coplanar with $\hat{\omega}_{1}$ and $\hat{\omega}_{2}$ (i.e., in the span of $\hat{\boldsymbol{\omega}}_{1}$ and $\hat{\boldsymbol{\omega}}_{2}$ ) would have resulted in a valid position $\mathbf{r}_{m}$ for the dipole, so we are left with one degree of freedom (i.e., rotation of $\hat{\boldsymbol{\Omega}}$ in the plane) to choose $\hat{\boldsymbol{\Omega}}$ to result in a desirable location of $\mathbf{r}_{m}$ based on some additional optimization criterion.

2) $\hat{\boldsymbol{\omega}}_{1}, \hat{\boldsymbol{\omega}}_{2}$, and $\mathbf{d}$ not coplanar: Next we consider the more complicated case where $\hat{\boldsymbol{\omega}}_{1}$ and $\hat{\boldsymbol{\omega}}_{2}$ are not coplanar with their displacement vector, which is the only fully threedimensional case considered so far. In general, neither $\mathbf{r}_{m}$ nor $\hat{\Omega}$ is known, so the search space for a potential solution is five-dimensional. We will show in this section that the problem's physical constraints can be used to reformulate the search for a solution to a two-dimensional search space, which can tractably be solved online using existing numerical techniques.

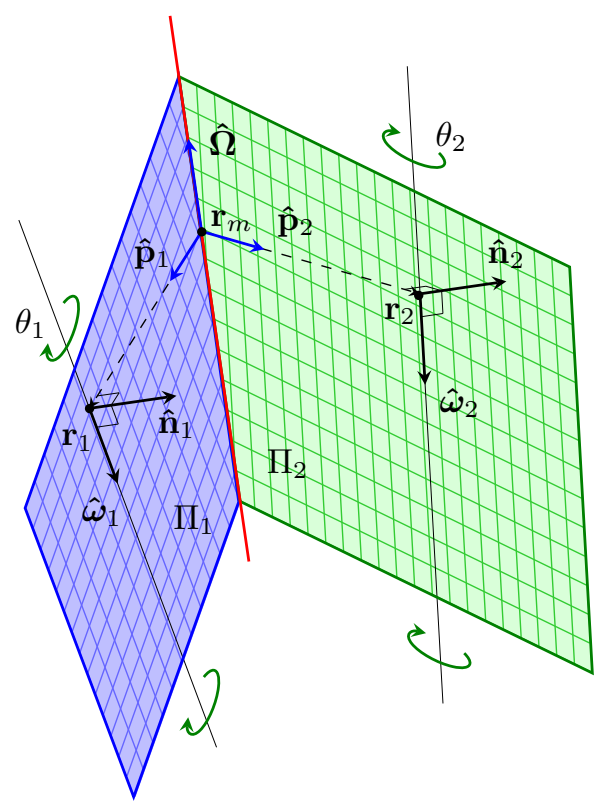

Fig. 2. Geometry used in Sec. II-C.2.

From (2), we know that $\hat{\boldsymbol{\omega}}_{1}, \hat{\mathbf{p}}_{1}$, and $\hat{\boldsymbol{\Omega}}$ must be coplanar, and that $\hat{\boldsymbol{\omega}}_{2}, \hat{\mathbf{p}}_{2}$, and $\hat{\boldsymbol{\Omega}}$ must be coplanar, but the two planes need not be the same. Consider the vectors $\hat{\boldsymbol{\omega}}_{1}, \hat{\mathbf{p}}_{1}$, and $\hat{\boldsymbol{\Omega}}$ to lie in a plane $\Pi_{1}$, and the vectors $\hat{\boldsymbol{\omega}}_{2}, \hat{\mathbf{p}}_{2}$, and $\hat{\boldsymbol{\Omega}}$ to lie in a plane $\Pi_{2}$, as depicted in Fig. 2. A plane is defined as the set of all points $\mathbf{r}$ in $\mathbb{R}^{3}$ that satisfy the equation

$$
\mathbf{r} \cdot \hat{\mathbf{n}}=\mathbf{r}_{p} \cdot \hat{\mathbf{n}} \equiv h,
$$

where $\hat{\mathbf{n}}$ is the plane unit normal, and $\mathbf{r}_{p}$ is any known point on the plane. In order to satisfy both coplanarity constraints, the vector $\hat{\Omega}$ must exist at the intersection of the two planes $\Pi_{1}$ and $\Pi_{2}$. The equation defining the line of intersection is

$$
\mathbf{L}_{\Pi_{1}, \Pi_{2}}(\lambda)=\alpha_{1} \hat{\mathbf{n}}_{1}+\alpha_{2} \hat{\mathbf{n}}_{2}+\lambda\left(\hat{\mathbf{n}}_{1} \times \hat{\mathbf{n}}_{2}\right),
$$

where

$$
\alpha_{1}=\frac{h_{1}-h_{2} \hat{\mathbf{n}}_{1} \cdot \hat{\mathbf{n}}_{2}}{1-\left(\hat{\mathbf{n}}_{1} \cdot \hat{\mathbf{n}}_{2}\right)^{2}}, \quad \alpha_{2}=\frac{h_{2}-h_{1} \hat{\mathbf{n}}_{1} \cdot \hat{\mathbf{n}}_{2}}{1-\left(\hat{\mathbf{n}}_{1} \cdot \hat{\mathbf{n}}_{2}\right)^{2}},
$$

and $\lambda \in \mathbb{R}$ parameterizes a point on the line [20], and $\hat{\boldsymbol{\Omega}}$ is

$$
\hat{\mathbf{\Omega}}=\frac{\hat{\mathbf{n}}_{1} \times \hat{\mathbf{n}}_{2}}{\left\|\hat{\mathbf{n}}_{1} \times \hat{\mathbf{n}}_{2}\right\|},
$$

Note that the negative of the solution in (10) generates rotating fields about the correct axes, but with the opposite sense from that desired.

The orientation of a plane is defined by its unit normal. We can consider each plane $\Pi_{i}$ as being rotated by some angle $\theta_{i}$ about $\hat{\boldsymbol{\omega}}_{i}$ from an initial vector $\hat{\mathbf{n}}_{0}$. We know that $\hat{\boldsymbol{\omega}}_{1}$ is orthogonal to $\hat{\mathbf{n}}_{1}$ since $\hat{\boldsymbol{\omega}}_{1}$ lies in the plane $\Pi_{1}$, and similarly $\hat{\boldsymbol{\omega}}_{2}$ is orthogonal to $\hat{\mathbf{n}}_{2}$. The choice of $\hat{\mathbf{n}}_{0}$ should be mutually orthogonal to $\hat{\boldsymbol{\omega}}_{1}$ and $\hat{\boldsymbol{\omega}}_{2}$, and since we are considering the case where $\hat{\boldsymbol{\omega}}_{1} \neq \hat{\boldsymbol{\omega}}_{2}, \hat{\mathbf{n}}_{0}$ can always be calculated as

$$
\hat{\mathbf{n}}_{0}=\frac{\hat{\boldsymbol{\omega}}_{1} \times \hat{\boldsymbol{\omega}}_{2}}{\left\|\hat{\boldsymbol{\omega}}_{1} \times \hat{\boldsymbol{\omega}}_{2}\right\|} .
$$


Using Rodrigues' formula [21], a given plane's normal can be described as a function of $\theta_{i}$ :

$$
\begin{aligned}
\hat{\mathbf{n}}_{i}=\hat{\mathbf{n}}_{0} & +\left(\hat{\boldsymbol{\omega}}_{i} \times \hat{\mathbf{n}}_{0}\right) \sin \theta_{i} \\
& +\hat{\boldsymbol{\omega}}_{i} \times\left(\hat{\boldsymbol{\omega}}_{i} \times \hat{\mathbf{n}}_{0}\right)\left(1-\cos \theta_{i}\right) .
\end{aligned}
$$

Thus, given values of $\theta_{1}$ and $\theta_{2}$, the plane normals can be found using (12), and $\hat{\Omega}$ can then be found using (10). These values for $\theta_{1}$ and $\theta_{2}$ will be the two indepedent parameters that we will use in our numerical search for our solution, so for the remainder of the analysis we assume that $\hat{\Omega}, \hat{\mathbf{n}}_{\mathbf{1}}$, and $\hat{\mathbf{n}}_{\mathbf{2}}$ are known.

To find the necessary dipole position $\mathbf{r}_{m}$, we can find the point of intersection between two lines in space: the line along $\mathbf{p}_{1}\left(\mathbf{L}_{1}\right)$ and the line of intersection between the two planes $\left(\mathbf{L}_{\Pi_{1}, \Pi_{2}}\right.$, defined in (8)). Given two noncoincidental points $\mathbf{x}_{1}=\mathbf{r}_{1}$ and $\mathbf{x}_{2}=\mathbf{r}_{1}-\hat{\mathbf{p}}_{1}$ on $\mathbf{L}_{1}$ and two non-coincidental points $\mathbf{x}_{3}=\alpha_{1} \hat{\mathbf{n}}_{1}+\alpha_{2} \hat{\mathbf{n}}_{2}$ and $\mathbf{x}_{4}=\alpha_{1} \hat{\mathbf{n}}_{1}+\alpha_{2} \hat{\mathbf{n}}_{2}+\left(\hat{\mathbf{n}}_{1} \times \hat{\mathbf{n}}_{2}\right)$ on $\mathbf{L}_{\Pi_{1}, \Pi_{2}}$, define the vector quantities $\mathbf{a}=\mathbf{x}_{2}-\mathbf{x}_{1}, \mathbf{b}=\mathbf{x}_{4}-\mathbf{x}_{3}$, and $\mathbf{c}=\mathbf{x}_{3}-\mathbf{x}_{1}$. From [22], the point of intersection of two non-skew lines in three-dimensional space as parameterized above is

$$
\mathbf{x}_{i}=\mathbf{x}_{1}+\left(\frac{(\mathbf{c} \times \mathbf{b}) \cdot(\mathbf{a} \times \mathbf{b})}{\|\mathbf{a} \times \mathbf{b}\|^{2}}\right) \mathbf{a} .
$$

Substituting the plane equations (7) and other known quantities into (13), the dipole position is calculated as

$$
\mathbf{r}_{m}=\mathbf{r}_{1}+\left(\frac{\mathbf{d} \cdot \hat{\mathbf{n}}_{2}}{\hat{\mathbf{p}}_{1} \cdot \hat{\mathbf{n}}_{2}}\right) \hat{\mathbf{p}}_{\mathbf{1}} .
$$

The solution process is structured as follows. For a given set of $\theta_{1}$ and $\theta_{2},(12)$ is used to compute $\hat{\mathbf{n}}_{1}$ and $\hat{\mathbf{n}}_{2},(10)$ is used to compute $\hat{\Omega}$, (4)-(6) are used to compute $\hat{\mathbf{p}}_{1}$ for the given $\hat{\boldsymbol{\omega}}_{1}$, and (14) is used to compute $\mathbf{r}_{m}$. Then, since $\mathbf{r}_{m}=\mathbf{r}_{2}-\mathbf{p}_{2}$, the unit vector $\hat{\mathbf{p}}_{2}$ can be found. Using $\hat{\mathbf{p}}_{2}$ and (3), $\hat{\boldsymbol{\omega}}_{2}^{\prime}$ can be computed at $\mathbf{r}_{2}$, which in general will not be the same $\hat{\omega}_{2}$ as desired. All that remains is to solve for the required values of $\theta_{1}$ and $\theta_{2}$ that will give the desired $\hat{\omega}_{2}$ at $\mathbf{r}_{2}$. In general, there may not be a closed-form solution for $\theta_{1}$ and $\theta_{2}$. In practice, it may be necessary to find the solutions numerically. Since we use $\hat{\omega}_{1}$ to find $\hat{\mathbf{p}}_{1}$, we know that we will always generate the desired $\hat{\omega}_{1}$. Therefore, we define an error term

$$
E\left(\theta_{1}, \theta_{2}\right)=\left\|\hat{\boldsymbol{\omega}}_{2}-\hat{\boldsymbol{\omega}}_{2}^{\prime}\left(\theta_{1}, \theta_{2}\right)\right\| .
$$

Since it is known that the minimum value of $E$ is 0 , which occurs when $\hat{\boldsymbol{\omega}}_{2}=\hat{\boldsymbol{\omega}}_{2}^{\prime}$, we can use a two-dimensional local search method such as hill climbing, gradient descent, or simulated annealing to find values of $\theta_{i}$ that result in an error $E=0$ [23].

We find that, in this most general case, the positions $\mathbf{r}_{m}$ lie on a closed curve in space, with a unique rotation axis $\hat{\Omega}$ at each point on the curve. An example of a set of $\mathbf{r}_{m}$ and corresponding $\hat{\boldsymbol{\Omega}}$ for the case of $\mathbf{r}_{1}=[4,1,2]^{\top}$, $\mathbf{r}_{2}=[2,4,4]^{\mathrm{\top}}, \hat{\boldsymbol{\omega}}_{1}=[-0.179,0.248,-0.952]^{\top}$, and $\hat{\boldsymbol{\omega}}_{2}=$ $[0.231,0.308,-0.923]^{\top}$ is shown in Fig. 3. Note that this is the same example depicted in Fig. 2. In practice, one solution $\mathbf{r}_{m}$ and $\hat{\boldsymbol{\Omega}}$ is selected as the optimal solution for some cost function designed to solve a particular problem. For instance, $\mathbf{r}_{m}$ could be selected to yield equal magnetic field strength at both desired locations, to affect the net magnetic force on each of the UMDs as they are actuated by their respective nonuniform rotating magnetic fields [24], or even to avoid collisions of the dipole source with obstacles. The location of $\mathbf{r}_{m}$ in Fig. 3 represents a location that is equidistant from $\mathbf{r}_{1}$ and $\mathbf{r}_{2}$; this example shows that one of two possible solutions was found for such a cost function, based on the initial guess.

\section{Solution Algorithm}

Alg. 1 describes the complete method to find a valid solution to generate two independent rotating magnetic fields with a single rotating dipole. The algorithm does not include optimizing the solution for a specific application.

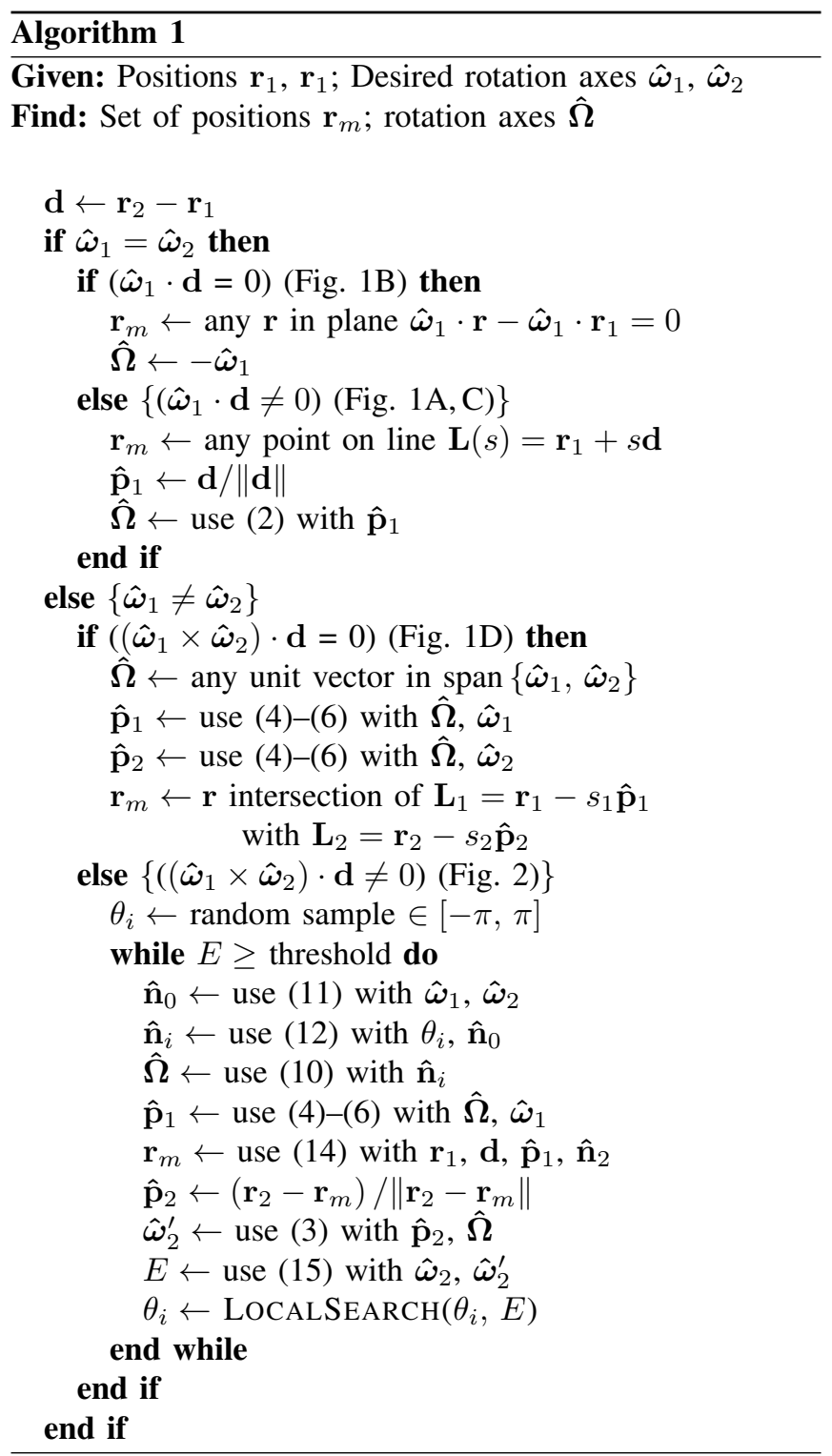




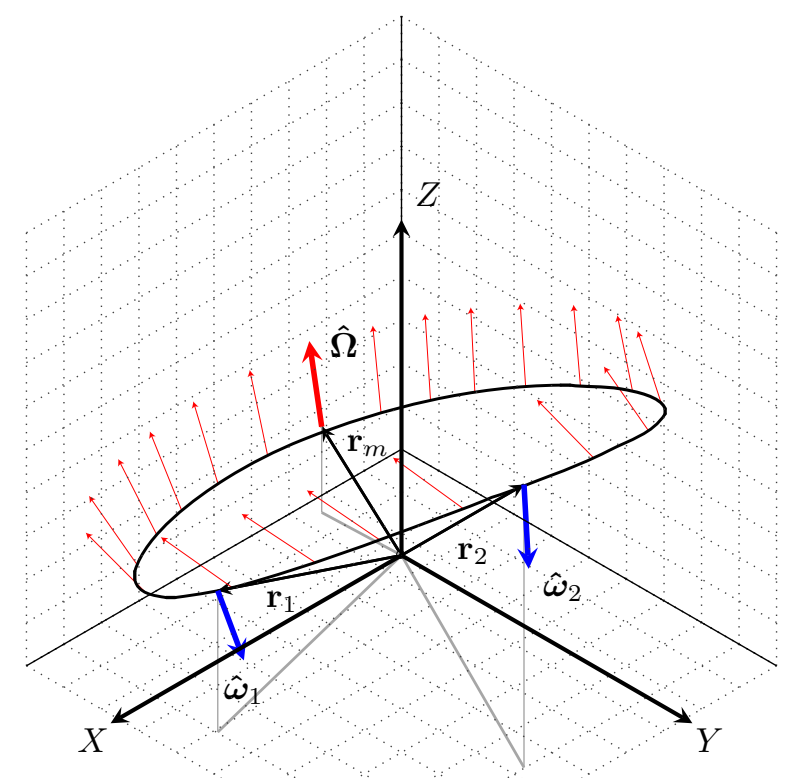

Fig. 3. Example of closed curve of numerically solved positions $\mathbf{r}_{m}$ for the case where $\mathbf{r}_{1}=[4,1,2]^{\top}, \mathbf{r}_{2}=[2,4,4]^{\top}, \hat{\boldsymbol{\omega}}_{1}=$ $[-0.179,0.248,-0.952]^{\top}$, and $\hat{\boldsymbol{\omega}}_{2}=[0.231,0.308,-0.923]^{\top}$.

\section{EXPERIMENTS}

We now seek to experimentally demonstrate the method in a real application. Since this is only a proof of concept, we shall consider the control of two magnetic screws within a block of agar gel as one possible example of the UMDs to which this method can be applied. Despite the problems of non-ideal behavior of magnetic screws observed within agar gel in previous work [6], we choose this application to avoid the effects of gravity and mitigate magnetic interaction between the UMDs. Although accurate steering and pathplanning of magnetic screws through soft tissue and phantoms like agar gel is still an open problem, if the screws are operated slow enough and not steered too hard, then barring local disturbances and errors in initial placement and orientation, the screws can be driven in an open-loop fashion.

The magnetic screws and agar gel are made in the same manner as outlined in [7]. Two standard pan-head No.-0 screws made of 18-8 stainless steel were chosen for their non-ferromagnetic properties and small size. The heads are removed and a $1 \mathrm{~mm} \times 1 \mathrm{~mm} \times 1 \mathrm{~mm}$ N50-grade $\mathrm{NdFeB}$ permanent magnet with a magnet dipole moment of $0.0011 \mathrm{~A} \cdot \mathrm{m}^{2}$ is affixed with the magnet dipole orthogonal to the screw body axis. The screws, shown in Fig. $4 \mathrm{a}$, are $5.6 \mathrm{~mm}$ in length, $1.5 \mathrm{~mm}$ in diameter, and with a thread pitch of $0.32 \mathrm{~mm}$. The screws are placed within $101.6 \mathrm{~mm} \times 101.6 \mathrm{~mm} \times 25.4 \mathrm{~mm}$ blocks of $0.5 \mathrm{wt} \%$ agar gel, shown in Fig. 4b. The screws are placed in position by hand as close to horizontal as possible.

The screws are actuated using a new device developed in our lab, the spherical-actuator-magnet manipulator (SAMM),

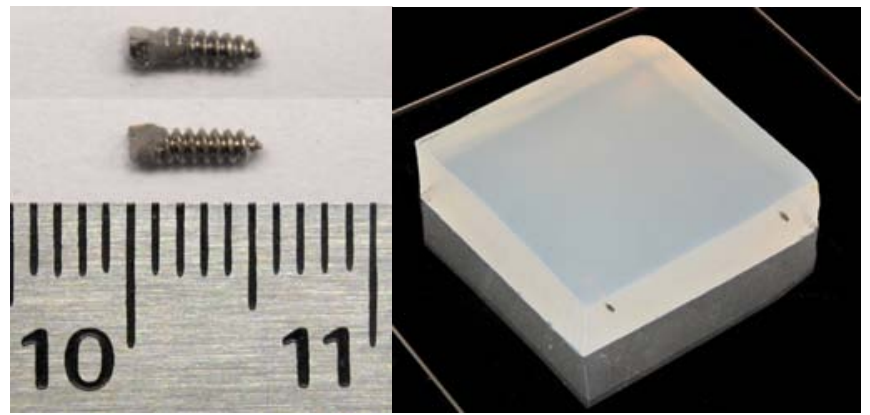

(a)

(b)

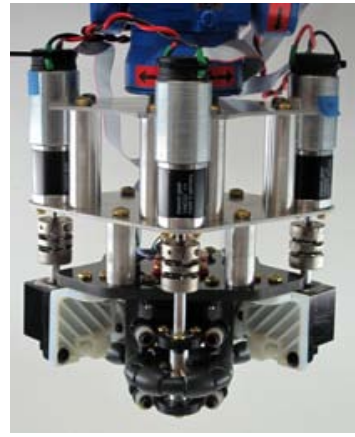

(c)

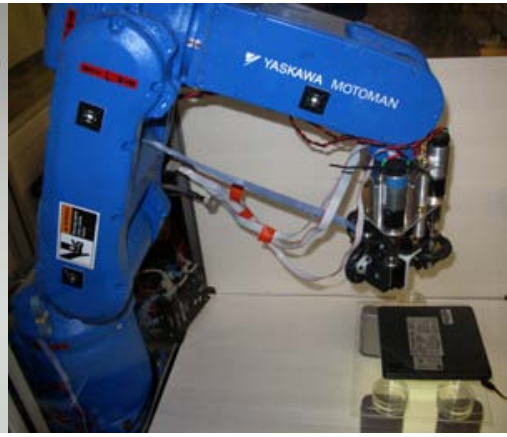

(d)
Fig. 4. (a) Two screws used in experiments made of 18-8 stainless steel and affixed with a $1 \mathrm{~mm}$ cubic permament magnet. (b) Screws run in agar gel cut into $101.6 \mathrm{~mm} \times 101.6 \mathrm{~mm} \times 25.4 \mathrm{~mm}$ blocks. (c) The spherical-actuatormagnet manipulator (SAMM) employs orthogonally mounted omniwheels to rotate a spherical permanent magnet about any desired axis, independent of the position of the magnet [25]. (d) The Yaskawa Motoman MH5 6DOF robotic manipulator fitted with the SAMM, in the complete experiment setup.

which acts as a magnetic end-effector of a Yaskawa Motoman MH5 6-DOF robotic manipulator (Fig. 4c and 4d).

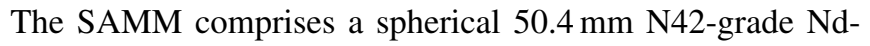
$\mathrm{FeB}$ permanent magnet with a magnet dipole moment of $71.6 \mathrm{~A} \cdot \mathrm{m}^{2}$ that can be rotated about any axis [25]. Within the workspace of the robot manipulator, we constructed a simple enclosure that enabled a Canon PowerShot G10 to record videos from beneath the agar gel block, which was lit from above by a fluorescent lamp.

Two different simple screw trajectories were designed for this demonstration: one to separate the screws from an initial separation of $31.8 \mathrm{~mm}$ to $76.2 \mathrm{~mm}$ (divergent trajectory), and one to bring the screws together from a separation of $76.2 \mathrm{~mm}$ to $31.8 \mathrm{~mm}$ (convergent trajectory). From the set of $\mathbf{r}_{m}$ and $\hat{\boldsymbol{\Omega}}$ found using Alg. 1, the position equidistant to each screw was selected at each time step. For each run, the spherical magnet was rotated at a constant speed of $0.58 \mathrm{~Hz}$, which was chosen sufficiently slow to maintain synchronization with the screws as they travel in the agar block.

The resulting experimental runs are shown in the attached video. The divergent trajectory is shown in Fig. 5a. The odd behavior observed in the lower screw around the $70 \mathrm{sec}$ mark is when that screw moved downward (i.e., out of the 


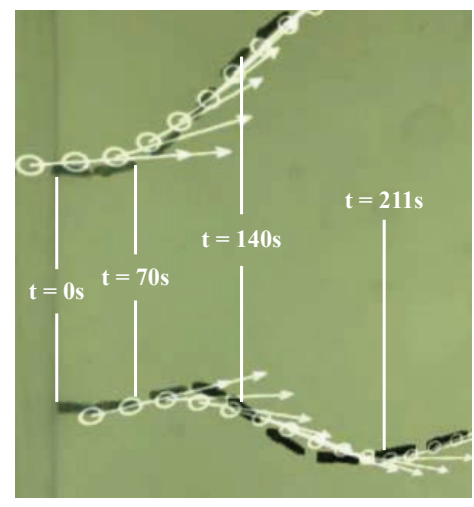

(a)

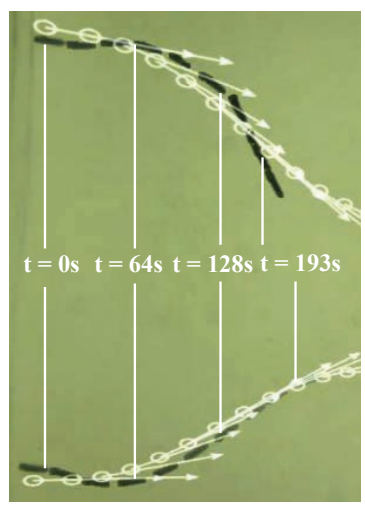

(b)
Fig. 5. Experimental runs demonstrating proof of concept, also shown in the attached video. (a) Divergent trajectory. (b) Convergent trajectory.

page) into the agar gel before diverging. This may be due to local disturbances as the screw traverses the agar, and may also be due to undesirable, but previously observed, out-of-plane motion as well as sensitivity to the placement of the screws relative to the spherical magnet [6]. The convergent trajectory is shown in Fig. 5b, which more closely followed the intended open-loop path. As in the divergent case, it is clear that both screws are being influenced by the independent rotating magnetic fields beyond what could be expected using rotating uniform magnet fields for propulsion.

\section{COnclusions And Future Work}

This paper has extended and built upon the previous work in magnetic actuation by proposing a method to generate two independent rotating magnetic fields using a single magnet dipole. We have developed an algorithmic approach to solving both special cases when the desired axes of rotation are equal, as well as the general cases when the rotation axes are not equal. Furthermore, we have demonstrated that two screws within an agar gel environment can be made to move apart or be brought together in a purposeful manner. Future work should include exploring whether an analytical solution exists, how to optimally choose a solution for a given problem, and path-planning methods that account for both UMDs and the magnet actuator.

\section{ACKNOWLEDGMENT}

The authors would like to thank Arthur Mahoney, Andrew Petruska, and Lisandro Leon for fruitful discussions during the development of this material.

\section{REFERENCES}

[1] B. J. Nelson, I. K. Kaliakatsos, and J. J. Abbott, "Microrobots for minimally invasive medicine," Annual Review of Biomedical Engineering, vol. 12, pp. 55-85, 2010.

[2] A. Ghosh and P. Fischer, "Controlled propulsion of artificial magnetic nanostructured propellers." Nano Letters, vol. 9, no. 6, pp. 2243-2245, 2009.

[3] L. Zhang, J. J. Abbott, L. Dong, B. E. Kratochvil, D. Bell, and B. J. Nelson, "Artificial bacterial flagella: Fabrication and magnetic control," Applied Physics Letters, vol. 94, no. 6, p. 064107, 2009.
[4] K. E. Peyer, L. Zhang, and B. J. Nelson, "Bio-inspired magnetic swimming microrobots for biomedical applications," Nanoscale, vol. 5, no. 4, pp. 1259-1272, 2013.

[5] K. Ishiyama, M. Sendoh, and K. I. Arai, "Magnetic micromachines for medical applications," Journal of Magnetism and Magnetic Materials, vol. 242, pp. 41-46, 2002.

[6] A. W. Mahoney, N. D. Nelson, E. M. Parsons, and J. J. Abbott, "Non-ideal behaviors of magnetically driven screws in soft tissue," in Intelligent Robots and Systems, IEEE/RSJ International Conference on, 2012, pp. 3559-3564.

[7] N. D. Nelson, J. Delacenserie, and J. J. Abbott, "An empirical study of the role of magnetic, geometric, and tissue properties on the turning radius of magnetically driven screws," in Robotics and Automation, IEEE International Conference on, 2013, pp. 5372-5377.

[8] C. Hu, W. Yang, D. Chen, M. Q.-H. Meng, and H. Dai, "An improved magnetic localization and orientation algorithm for wireless capsule endoscope," in Engineering in Medicine and Biology Society, International Conference of the IEEE, 2008, pp. 2055-2058.

[9] M. Gao, C. Hu, Z. Chen, H. Zhang, and S. Liu, "Design and fabrication of a magnetic propulsion system for self-propelled capsule endoscope," Biomedical Engineering, IEEE Transactions on, vol. 57, no. 12, pp. 2891-2902, 2010.

[10] J. J. Abbott, K. E. Peyer, M. Cosentino Lagomarsino, L. Zhang, L. Dong, I. K. Kaliakatsos, and B. J. Nelson, "How should microrobots swim?" The International Journal of Robotics Research, vol. 28, no. 11-12, 2009.

[11] A. W. Mahoney and J. J. Abbott, "Generating rotating magnetic fields with a single permanent magnet for propulsion of untethered magnetic devices in a lumen," IEEE Transactions on Robotics, vol. 30, no. 2, pp. 411-420, 2014.

[12] A. J. Petruska and J. J. Abbott, "Omnimagnet: An omnidirectional electromagnet for controlled dipole-field generation," IEEE Transactions on Magnetics, vol. 50, no. 7, pp. 1-10, 2014.

[13] W. Zhang, P. Huang, and Y.-G. Meng, "Mechanism and experiment research on rotational magnetic field generated by circumferentially arrayed permanent magnets," Journal of Engineering Design, vol. 3, pp. 2367-2372, 2008.

[14] S. Floyd, E. Diller, C. Pawashe, and M. Sitti, "Control methodologies for a heterogeneous group of untethered magnetic micro-robots," The International Journal of Robotics Research, vol. 30, no. 13, pp. 1553$1565,2011$.

[15] E. Diller, S. Miyashita, and M. Sitti, "Remotely addressable magnetic composite micropumps," RSC Advances, vol. 2, no. 9, pp. 3850-3856, 2012.

[16] E. Diller, J. Giltinan, and M. Sitti, "Independent control of multiple magnetic microrobots in three dimensions," The International Journal of Robotics Research, vol. 32, no. 5, 2013.

[17] A. W. Mahoney, N. D. Nelson, K. E. Peyer, B. J. Nelson, and J. J. Abbott, "Behavior of rotating magnetic microrobots above the step-out frequency with application to control of multi-microrobot systems," Applied Physics Letters, vol. 104, no. 14, p. 144101, 2014.

[18] T. W. R. Fountain, P. V. Kailat, and J. J. Abbott, "Wireless control of magnetic helical microrobots using a rotating-permanent-magnet manipulator," in Robotics and Automation, IEEE International Conference on, 2010, pp. 576-581.

[19] A. J. Petruska and J. J. Abbott, "Optimal permanent-magnet geometries for dipole field approximation," Magnetics, IEEE Transactions on, vol. 49, no. 2, pp. 811-819, 2013.

[20] J. Vince, Geometry for Computer Graphics. Springer, 2005.

[21] R. M. Murray, Z. Li, and S. S. Sastry, A Mathematical Introduction to Robotic Manipulation. CRC press, 1994.

[22] R. Goldman, Graphics Gems, A. S. Glassner, Ed. Academic Press, Inc., 1990.

[23] S. Russel and P. Norvig, Artificial Intelligence: A Modern Approach. Prentice Hall, 2003.

[24] A. W. Mahoney, S. E. Wright, and J. J. Abbott, "Managing the attractive magnetic force between an untethered magnetically actuated tool and a rotating permanent magnet," in Robotics and Automation, IEEE International Conference on, 2013, pp. 5366-5371.

[25] S. E. Wright, A. W. Mahoney, K. M. Popek, and J. J. Abbott, "A spherical-magnet end-effector for robotic magnetic manipulation," in Robotics and Automation, IEEE International Conference on, 2015. 